\title{
Glanzmann's thrombasthenia: a rare bleeding disorder in a Nigerian girl
}

\author{
Osita U Ezenwosu, ${ }^{1}$ Barth F Chukwu, ${ }^{1}$ Ndubuisi A Uwaezuoke, ${ }^{1}$ Ifeyinwa
} L Ezenwosu, ${ }^{2}$ Anthony N Ikefuna, ${ }^{1}$ Ifeoma J Emodi ${ }^{1}$

1. Department of Paediatrics, College of Medicine, University of Nigeria, Enugu Campus, Nigeria.

2. Department of Community Medicine, University of Nigeria Teaching Hospital, Enugu, Nigeria.

\section{Emails:}

Barth Chukwu: chizzy_bath@yahoo.com; Ndubuisi Uwaezuoke: nduwaezuoke@gmail.com; Ifeyinwa Ezenwosu: doclily17@yahoo.com; Anthony Ikefuna: nnaaikefuna@yahoo.com; Ifeoma Emodi: ifeoma_emodi@yahoo.com

\begin{abstract}
Introduction: Glanzmann's Thrombasthenia (GT) is a rare autosomal recessive bleeding disorder due to defective platelet membrane glycoprotein GP IIb/IIIa (integrin $\alpha \mathrm{IIb} \beta 3$ ). The prevalence is estimated at 1:1,000,000 and it is commonly seen in areas where consanguinity is high.

Case Presentation: The authors report a 12 year old Nigerian girl of Igbo ethnic group, born of non-consanguineous parents, who presented with prolonged heavy menstrual bleeding which started at menarche 3 months earlier, weakness and dizziness. She had a past history of recurrent episodes of prolonged epistaxis, gastrointestinal bleeding and gum bleeding during early childhood. On examination, she was severely pale with a haemic murmur and vaginal bleeding. The initial diagnosis was menorrhagia secondary to bleeding diathesis possibly von Willebrand's Disease. She was on supportive treatment with fresh whole blood, fresh frozen plasma and platelets until diagnosis of GT was made in the USA. Currently, she is on 3 monthly intramuscular Depo-provera with remarkable improvement.

Conclusion: To the best of our knowledge, this is the first documented report of GT in our environment where consanguinity is rarely practised. Our health facilities require adequate diagnostic and treatment facilities for rare diseases like GT.

Keywords: Glanzmann's thrombasthenia, Menorrhagia, Nigerian girl.

DOI: https://doi.org/10.4314/ahs.v20i2.27

Cite as: Ezenwosu OU, Chukwn BF, Uwaezuoke NA, Ezenwosu IL, Ikefuna AN, Emodi IJ. Glanzmann's thrombasthenia: a rare bleeding disorder in a Nigerian girl. Afri Health Sci. 2020; 20(2): 753-757. bttps:// doi.org/10.4314/ abs.v20i2.27
\end{abstract}

\section{Introduction}

Glanzmann's Thrombasthenia (GT) is an autosomal recessive bleeding disorder due to deficiency or abnormality in the platelet membrane glycoproteins IIb or IIIa (GPIIb/IIIa) - currently known as integrin $\alpha \operatorname{IIb} \beta 3 .{ }^{1,2}$ This integrin is found in abundance on the platelet membrane with the major function of mediating platelet aggregation and firm adhesion of platelets to the subendothelial matrix. ${ }^{2}$ Defective platelet integ-
Corresponding author:
Osita U Ezenwosu,
Department of Paediatrics,
College of Medicine,
University of Nigeria Enugu Campus, Nigeria.
Phone number: +234-803-6679-730
Email: osita.ezenwosu@unn.edu.ng

rin, therefore, results in impaired platelet-platelet interaction and defective clot formation leading to abnormal bleeding. ${ }^{1,3}$

The prevalence of GT is unknown but it is estimated at 1:1 million with a slight female preponderance.4 It has been noted to be commonly seen in areas where consanguinity is high or practiced.5 The typical clinical presentation include mucocutaneous bleeding (purpura, epistaxis, gingival haemorrhage), gastro-intestinal bleeding and heavy menstrual bleeding. ${ }^{1-5}$

It is important to diagnose GT and differentiate it from other inherited bleeding disorders and similar platelet function abnormalities. ${ }^{6}$ Aside the typical clinical presentations, laboratory investigations show normal platelet count- with presumably normal size, prolonged skin bleeding time, prolonged Platelet Function Analyzer 100 (PFA-100) closure times, impaired or absent platelet aggregation response to all agonists (ADP, thrombin, epinephrine, collagen, ARA) except ristocetin. ${ }^{1,3,4}$ 
The diagnosis of GT is confirmed with immunophenotyping analysis using flow cytometry which shows abnormal antibody binding to the GPIIb/IIIa. ${ }^{1,3,4}$

Although bleeding problems can be severe in patients with GT, with appropriate and careful supportive care the prognosis for normal survival and an active life remains very good. ${ }^{5,7}$

We report a case of 12 year old Nigerian girl with GT. The need for awareness, provision of adequate diagnostic and treatment modalities in our health facilities for better management of this rare condition is highlighted.

\section{Case report}

O.C. is a 12 year old female who presented with prolonged heavy menstrual flow of three months, associated weakness and dizziness of one week. The heavy menstrual bleeding was noticed on achieving menarche three months earlier and had been recurrent. There was no bleeding from any other part of the body. She had past history of recurrent episodes of prolonged bleeding ranging from epistaxis, upper gastro-intestinal bleeding (coffee-coloured vomitus) to prolonged gum bleeds (lasting more than one hour). Gum bleeding followed tooth erruption and was noticed from age of 20 months to early childhood. Episodes of epistaxis lasted for about 15-20 minutes and abated with nasal clamping and cotton wool packs. These episodes of bleeding were managed conservatively in our centre up to the age of 3 years when she was lost to follow-up and re-presented with the above complaints. There was a history of prolonged nose bleeding in the mother and maternal aunt until adulthood though they were not investigated. Her mother had 28 days regular menstrual cycle with 5 days heavy flow which came in floods as she changed pads less than 3 hourly. She had three pregnancies and the birth histories were uneventful. Physical examination showed ill-looking child, severely pale with haemic murmur and vaginal bleeding. A diagnosis of menorrhagia secondary to bleeding diathesis possibly von Willebrand's Disease was made.
Investigations done included Full Blood Count (FBC) which showed severe anaemia $(\mathrm{Hb} 4.2 \mathrm{~g} / \mathrm{dl}$ ), normal WBC count $7,900 / \mathrm{mm} 3$, platelet count $200,000 / \mathrm{mm} 3$, Factor VIII $60 \mathrm{IU} / \mathrm{dL}$ (50-150 IU/dL), Factor IX 117.6 IU/dL (50-150 IU/dL), von Willebrand Ag $90.5 \mathrm{IU} / \mathrm{dL}$ (42-140 IU/dL), PT >12 seconds (control 13 seconds, INR 9.79), APTT 38 seconds (25.4-38.4 seconds), clotting time 30 minutes (4-8 minutes), bleeding time 20 minutes (2-7 minutes).

She initially received 2 units of fresh frozen plasma (FFP), 1 unit each of packed red cells and fresh whole blood following which her haemoglobin increased to $8.6 \mathrm{~g} / \mathrm{dl}$ with reduced bleeding. She received further supportive treatment with several units of fresh whole blood, FFP and platelet transfusions and later commenced on tranexamic acid 500mg tds and haematinics. However, due to persisting vaginal bleeding, the menstruation was suspended with regular intramuscular injection of Depo-provera.

Owing to limited resources to make definitive diagnosis, her parents requested for referral to the USA. At the Children's Hospital Egleston Atlanta, a definitive diagnosis of Glanzmann's Thrombasthenia was made by prolonged closure times with the PFA-100, normal platelet counts, platelet aggregometry (absent aggregation to thrombin, ADP, ATP, ARA but normal essentially with ristocetin) and flow cytometry (abnormal antibody binding to the GPIIb/IIIa) (Table 1). Recommendation from the hospital include recombinant factor 7 for severe bleeding with an option of platelet transfusion in our resource-limited setting; continued menstrual reduction with depo provera for heavy menstrual bleeding and anti-fibrinolytics (Aminocaproic acid or tranexamic acid) for mucosal bleeding.

On return to Nigeria, her menstrual flow was moderate volume and lasted for about 30 days. She has been on 3 monthly intramuscular Depo-provera (with prophylactic calcium lactate and vitamin D). Currently, there has been marked reduction in her menstrual flow to 6 days with normal $\mathrm{Hb}$ levels though she has few petechial rashes on the limbs and trunk. 


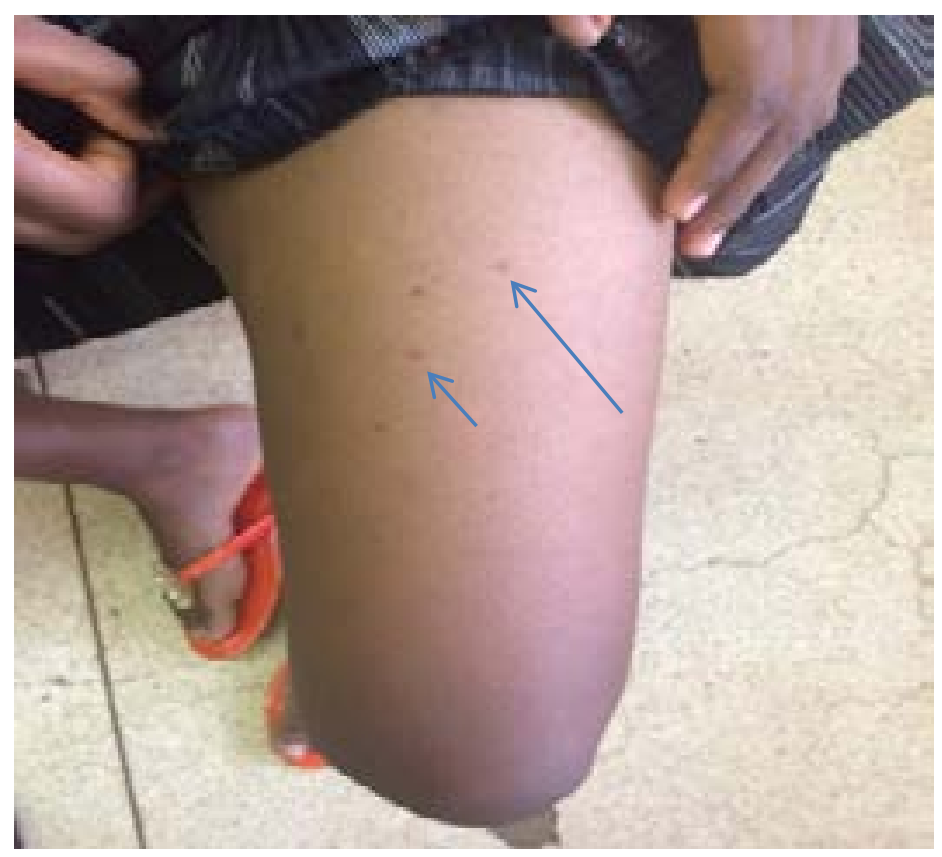

Figure 1: Arrows show few petechiae rashes on our patient's left thigh

Table 1: Flow Cytometry result from Children's Hospital Egleston Atlanta, USA

\author{
Platelet Glycoprotein Expression (Ib,IIb/IIIa) \\ Collected: $11 / 03 / 151635$ \\ Resulting lab: CHOA LAB \\ Value: (NOTE) \\ Comment: GPIb Monoclonal Antibody Binding Normal \\ GPIIb/IIIa Monoclonal Antibody Binding Abnormal \\ PGE Interpretation See Below
}

\title{
PGE Interpretation
}

Significantly reduced binding of monoclonal antibody to platelet glycoprotein IIb/IIIa is consistent with a clinical diagnosis of Glanzmann's Thrombasthenia. Platelets show normal monoclonal antibody binding to glycoprotein $\mathrm{Ib}$.

\section{Discussion}

Glanzmann's Thrombasthenia (GT) is a very rare autosomal recessive bleeding disorder with an estimated prevalence of $1: 1,000,000 .{ }^{4}$ The rarity and uneven geographic distribution of GT has been documented in an earlier review by George et $\mathrm{al}^{7}$ which noted that out of 177 patients, 12 were from the USA, 55 from Israel and Jordan, and 42 from India. Recent studies in Paki$\operatorname{stan}^{6,8}$ and Southern India9 also noted large numbers of patients with GT. The cause for this uneven distribution is the frequent occurrence of consanguineous marriage in these regions, allowing expression of autosomal recessive traits and suggesting a rare occurrence of asymptomatic heterozygous subjects. ${ }^{7}$ The association of GT with consanguinity is further emphasized by the observation that up to $90 \%$ of cases of GT in Pakistan ${ }^{8}$ and India ${ }^{9}$ were found in intermarriage settings. However, few cases have been reported from different 
parts of the world including Europe (France) ${ }^{10}$ Middle East (Saudi Arabia and UAE) $)^{11,12}$ and Africa (Tunisia). ${ }^{13}$ This is the first reported case from a location in Nigeria where consanguineous marriage is rare.

Although the age at presentation varies, majority of patients with GT present during early childhood and report frequent bleeding episodes through the first decade of life. ${ }^{3}$ George et $\mathrm{al}^{7}$ noted that most of the patients were diagnosed at less than 5 years while Irem and colleagues ${ }^{6}$ reported that $52.7 \%$ of their subjects manifested symptoms within the first year of life. This was also corroborated by Hejer and co-workers ${ }^{13}$ whose patient cohorts were diagnosed at age less than or equal to 4 years. Our index patient first presented at the age of 20 months. As reported, early childhood is the corresponding period of clinical expression of GT. ${ }^{14}$

At presentation, our patient manifested the typical features of GT with recurrent episodes of prolonged epistaxis, gum bleeds and upper gastro-intestinal bleeding. Epistaxis, gingival bleeding and purpura have been termed nearly constant features of $\mathrm{GT}^{5,7}$ and are the most reported worldwide. ${ }^{6-9,13}$ Another constant feature in the females, menorrhagia, which is worse during menarche is usually a challenge and may be particularly severe. ${ }^{3}$ Though we lost our patient to follow up, she re-presented at the age of 12 with prolonged heavy menstrual bleeding (HMB). Comprehensive screening of adolescent girls with HMB is important for identifying those with undiagnosed bleeding conditions such as GT, as accurate diagnosis might prevent potentially severe complications such as unnecessary surgical interventions. ${ }^{2}$ Additionally, in these females with GT, pregnancy carries multiple risks. To the pregnant females, the risks of abortions, antepartum and postpartum haemorrhages are all increased. ${ }^{15,16}$ The foetal risk is related to alloimmune thrombocytopenia (induced by transplacental passage of the maternal $\mathrm{IgG}$ anti-GP IIb/IIIa isoantibodies) and potential intracranial haemorrhage. ${ }^{15,16}$ In such cases, multidisciplinary care becomes paramount for ensuring optimal maternal and fetal outcomes. ${ }^{16}$

The screening tests PT, aPT'T levels, and platelet counts and morphology are normal in individuals with GT.1 The usual absent or delayed closure time on PFA-100 may be a preliminary indication of a platelet function disorder or Von Willebrand disease.1 However, platelet aggregometry is the gold standard for the diagnosis of GT which can be distinguished from other platelet function disorders by the lack of platelet aggregation in response to all agonists with a normal response to only ristocetin. ${ }^{1,6,9}$ Flow cytometry may be helpful in diagnosing GT, where low or absent GPIIb or GPIIIa (evidenced by abnormal antibody binding to the GPIIb/ IIIa) is characteristic. ${ }^{1,2,-6,13}$ Nevertheless, Flow cytometry studies may be normal in GT type 3 in which there is a qualitative defect in the GPIIb/IIIa receptor. Our patient had the initial screening tests in our centre but due to limited laboratory facilities to make definitive diagnosis, her diagnosis was made in the USA with PFA100 , platelet aggregometry and flow cytometry.

With appropriate and careful supportive care, the prognosis for GT remains very good. ${ }^{5,7}$ The main treatment option is the use of platelet concentrates transfusions to achieve haemostasis. ${ }^{2,4}$ To minimize risk of alloimmunisation following recurrent platelet transfusions, HLA-matched platelets are recommended treatment of choice but it may not be readily available in resource-limited settings. ${ }^{3-5}$ In those with heavy menstrual bleeding, menstrual reduction with Depo-Provera is a recommended treatment. The index patient is currently under our care and is doing well on Depo-Provera with reduced menstrual bleed.

\section{Conclusion}

To the best of our knowledge, this is the first documented report of GT in our environment where consanguinity is very rare. This calls for awareness and high index of suspicion of the condition in children with early bleeding disorders as well as females with menorrhagia who may be at risk of negative effect of GT on maternal and child outcomes during pregnancy.

Our health facilities require adequate diagnostic and treatment facilities for this rare diseases. Meanwhile, there is need for collaboration with centres in resource-rich countries where samples can be promptly analysed for diagnosis.

\section{Declaration of interest}

The authors declare no conflicts of interest.

\section{References}

1. Recht M, Chitlur M, Lam D, Sarnaik S, Rajpurkar M, Cooper DL, Gunawardena S. Epistaxis as a common presenting symptom of Glanzmann's Thrombasthenia , a rare qualitative platelet disorder: illustrative case examples. Case Reports in Emerg Med 2017; ID 8796425. https://doi.org/10.1155/2017/8796425.

2. Rajpurkar M, O’Brien SH, Haamid FW, Copper DL, Gunawardena S, Chitlur M. Heavy menstrual bleeding as a common presenting symptom of rare platelet dis- 
orders: illustrative case examples. Pediatr Adolesc Gynecol 2016; 29: 537-541 PubMed.

3. Alamelu J, Liesner R. Modern management of severe platelet function disorders. BrJ Haematol 2010; 149: 813-823 PubMed.

4. Diz-Kucukkaya R. Inherited platelet disorders including Glanzmann thrombasthenia and Bernard-Soulier syndrome. Hematol Am Soc Hematol Educ program 2013; 1: $268-275$.

5. Nurden AT. Glanzmann thrombasthenia. Orphanet J Rare Dis 2006; 1: 10-18 PubMed . http://www.OJRD. com/content/1/1/10.

6. Iqbal I, Farhan S, Ahmed N. Glanzmann thrombasthenia: a clinicopathological profile. J Coll Phys Surg Pakistan 2016; 26: 647-650.

7. George JN, Caen JP, Nurden AT. Glanzmann's throbasthenia: the spectrum of clinical disease. Blood 1990; 7: 1383-1395 PubMed.

8. Asif N, Zafar T, Hassan K, Ahmed S. Glanzmann's thrombasthenia. Ann Pak Inst Med Sci 2007; 3: 201-205. 9. Belurkar S, Kurien A, Singh PB. Glanzmann's thrombasthenia: the clinicopathological spectrum and its diversities. Ann Nigerian Med 2012; 6: 35-40 PubMed.

10. Bellucci S, Damaj G, Boval B, Rocha V, Devergie
A, Yacoub-Agha I et al. Bone marrow transplantation in severe Glanzmann thrombasthenia with antiplatelet alloimmunization. Bone marrow Transplantation 2000; 25: 327-330.

11. Alenzi MJ. Management of post-circumcision in Glanzmann's syndrome: case report and review of literatures. J Clin Case Reports 2016; 6: 688. doi:10.4172/21657920.1000688.

12. Al Wahab A, Nugud A, Nugud S, Alras Z. UnexplainedBleeding: Case Report of Glanzmann Thrombasthenia, MRSJ 2017. doi:10.15404/msrj/09.2017.0127. 13. Elmahmoudi H, Achour M, Belhedi N, Neji HB, Zahra K, Meddeb B et al. The Glanzmann's thrombasthenia in Tunisa: a cohort study. J Hematol 2017; 6: 44-48 PubMed.

14. Solh T, Botsford A, Solh M. Glanzmann's thrombasthenia: pathogenesis, diagnosis, and current and emerging treatment options. J Blood Med 2015; 6: 219-227. 15. Singh S, Swain S, Das L, Jena RK, Das OA, Sahoo B et al. Pregnancy in two patients of Glanzmann's thrombasthenia: a rare case report. Int J Reprod Contracept Obstet Gynaecol 2016; 5: 240-242.

16. Wijemanne A, Watt-Coote I, Austin S. Glanzmann thrombasthenia in pregnancy: optimizing maternal and fetal outcomes. Obstet Med 2016; 9: 169-170 PubMed. 